\title{
Power-Management Techniques for Wireless Sensor Networks and Similar Low-Power Communication Devices Based on Nonrechargeable Batteries
}

\author{
Agnelo Silva, ${ }^{1}$ Mingyan Liu, ${ }^{2}$ and Mahta Moghaddam ${ }^{1}$ \\ ${ }^{1}$ Department of Electrical Engineering/Electrophysics (EE), University of Southern California, Los Angeles, CA 90089, USA \\ ${ }^{2}$ Electrical Engineering and Computer Science (EECS), University of Michigan, Ann Arbor, MI 48109, USA \\ Correspondence should be addressed to Agnelo Silva, agnelors@gmail.com
}

Received 15 June 2012; Accepted 22 August 2012

Academic Editor: Hongxiang Li

Copyright (C) 2012 Agnelo Silva et al. This is an open access article distributed under the Creative Commons Attribution License, which permits unrestricted use, distribution, and reproduction in any medium, provided the original work is properly cited.

Despite the well-known advantages of communication solutions based on energy harvesting, there are scenarios where the absence of batteries (supercapacitor only) or the use of rechargeable batteries is not a realistic option. Therefore, the alternative is to extend as much as possible the lifetime of primary cells (nonrechargeable batteries). By assuming low duty-cycle applications, three powermanagement techniques are combined in a novel way to provide an efficient energy solution for wireless sensor networks nodes or similar communication devices powered by primary cells. Accordingly, a customized node is designed and long-term experiments in laboratory and outdoors are realized. Simulated and empirical results show that the battery lifetime can be drastically enhanced. However, two trade-offs are identified: a significant increase of both data latency and hardware/software complexity. Unattended nodes deployed in outdoors under extreme temperatures, buried sensors (underground communication), and nodes embedded in the structure of buildings, bridges, and roads are some of the target scenarios for this work. Part of the provided guidelines can be used to extend the battery lifetime of communication devices in general.

\section{Introduction}

Energy harvesting has been an intensive research area in wireless sensor networks (WSNs). However, for many important WSN scenarios, such energy option is not feasible, and specific power-management strategies are necessary for WSN nodes that are powered by nonrechargeable batteries. For instance, when extreme temperatures are involved, the charging process of rechargeable cells is strongly impacted, as empirically demonstrated in our work. The behavior observed in Figure 1 for a specific node was repeated by many others and the network was impacted for many periods of consecutive days.

Also, buried nodes used in wireless underground sensor networks [1] and nodes embedded inside the walls of buildings [2], in the roads, or in the internal structures of a bridge, typically cannot employ rechargeable cells. On the other hand, when nonrechargeable batteries (or primary cells) are considered for WSNs, a high operational cost is usually expected [3]. This is typically the case even for very low duty-cycle WSN applications. Two design areas can be exploited in order to increase the lifetime of a battery in this scenario: networking protocols and power management. The focus of this work is on the latter area. More specifically, after analyzing several current WSN node designs, we systematically identified three power management aspects that allow a significant extension of the battery lifetime.

After implementing a customized WSN solution following the techniques proposed in this work, we observed that the battery's lifetime can be enhanced to almost 300\% for some cases. More specifically, when the role of a WSN node is just taking and transmitting few measurements per day, the battery exchange will realistically occur closer to its age limit, typically between 5 to 10 years. With such results, a new generation of WSN applications for low-cost and unattended nodes becomes a reality. However, a trade-off to be considered is the increase on the high software/hardware complexity. Although the proposed techniques are tailored 


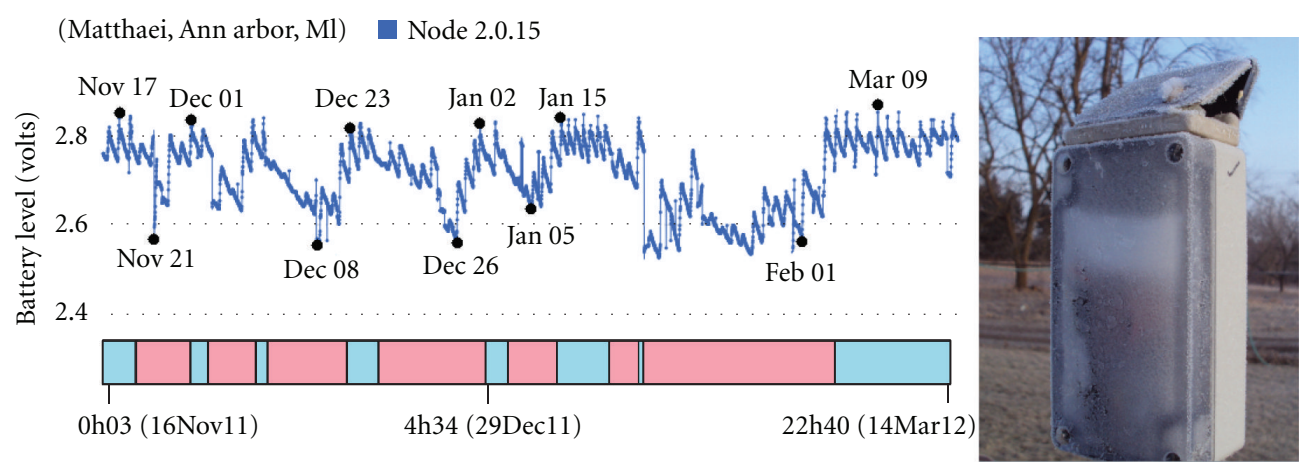

$\square$ Charging
$\square$ Not charging

FIGURE 1: Even with sufficient solar irradiation in some days, the efficiency of the recharging process of two NiMH cells (1.5 V nominal voltage each) is drastically impacted by extreme low temperatures $\left(\angle 0^{\circ} \mathrm{C}\right)$. Also, the efficiency of a solar panel, in particular a small one, is strongly impacted by snow and ice.

to some classes of WSN applications, some of the design principles in this work can still be considered for generic WSNs and ultra-low-power devices in general.

The rest of this paper is organized as follows. In Section 2, the power-gating technique is investigated for the WSN domain. In Section 3, another power-management strategy for WSN nodes powered by nonrechargeable batteries is presented: the avoidance of voltage regulators while the WSN node is sleeping. In Section 4, the design of a basic powermatching circuitry for WSN nodes, based on supercapacitors, is presented. Analytical and empirical results combining the 3 techniques are discussed. In Section 5, a comparison between our approach and traditional power-managment techniques is presented. The paper is concluded in Section 6.

\section{Power-Gating Technique}

In this section, the power-gating technique is compared with the conventional power-saving approach. Next, the novel concept of leakage gating is introduced as an extension of the power-gating technique for any interconnection line that can potentially allow leakage current while a module is inactive.

The power-gating technique applied to WSNs has been proposed as a way to save energy for both active and sleep modes of a device [4]. Such technique is basically the introduction of an electronic switch between an electronic module or chip and the power-supply line. Typically, a power gate is implemented by a PMOS or NMOS transistor for cutting the ground or power lines, respectively. Therefore, it is possible to temporarily shut down blocks of circuitries that are not in use. For instance, while the sensor node is taking measurements usually, the radio transceiver is not required and it can be turned off.

Although the majority of the modules in a WSN node have some sort of standby pin (sometimes called shutdown or power save), such control typically cannot effectively switch off all the internal circuitries of the module. In general, the quiescent current for the power-saving mode is small but still higher than $1 \mu \mathrm{A}$. The main reason why manufacturers usually do not adopt a power-gating solution is simple: using power-saving, the module can quickly return to normal operation as soon as the standby pin returns to disabled mode. However, if the delay caused by completely turning off/on a device (e.g., $1 \mathrm{~s}$ ) is not so critical for a given WSN application, an impressive energy reduction can be achieved by means of power-gating. This technique can be implemented externally to the module provided that the module does not have this functionality, as illustrated by the switches $\mathrm{SW}_{\mathrm{A}}, \mathrm{SW}_{\mathrm{B}}$, and $\mathrm{SW}_{\mathrm{C}}$ in Figure 5.

2.1. Simulation Analysis. To highlight the significant energy reduction achieved with the power-gating (PG) technique, a simple architecture for a WSN node is considered. This node is formed by a processor, a sensing module, and a radio transceiver. It periodically wakes up, performs some processing ( $1 \mathrm{~s})$, takes measurements $(5 \mathrm{~s})$, sends/receives data to/from the sink node $(3 \mathrm{~s})$, performs more processing $(1 \mathrm{~s})$, and finally sleeps again. Two scenarios are compared: (a) the power-saving technique, that is, the use of standby/sleep pins already available at the sensing and radio modules, and (b) the power-gating technique, implemented externally. It is assumed that the power source (e.g., batteries) is directly connected to the devices, that is, no additional loss due the existence of a voltage regulator or DC-DC converter. The values for the power consumed in each task/state are shown in Table 1. These values are typical ones based on off-theshelf analog switches and modules used in WSN nodes.

In this simulation, we compare the total energy consumption levels for a $24 \mathrm{~h}$ period when different application duty cycles (expressed as number of measurements per day) are considered, as shown in Figure 2. Note that, for the scope of this work, we disregard the network impact on the energy consumption. In Figure 2, the total energy consumption per day for the power-saving mode can be inferred as the sum 
TABLE 1: Typical power profile for a WSN node.

\begin{tabular}{lccc}
\hline & $\mathrm{MCU}$ & Sensors & Radio \\
\hline Active (regular operation) & $5 \mathrm{~mW}$ & $30 \mathrm{~mW}$ & $350 \mathrm{~mW}$ \\
Inactive (power saving) & $2 \mu \mathrm{W}$ & $5 \mu \mathrm{W}$ & $20 \mu \mathrm{W}$ \\
Inactive (power gating) & $2 \mu \mathrm{W}$ & $1 \mu \mathrm{W}$ & $1 \mu \mathrm{W}$ \\
\hline
\end{tabular}

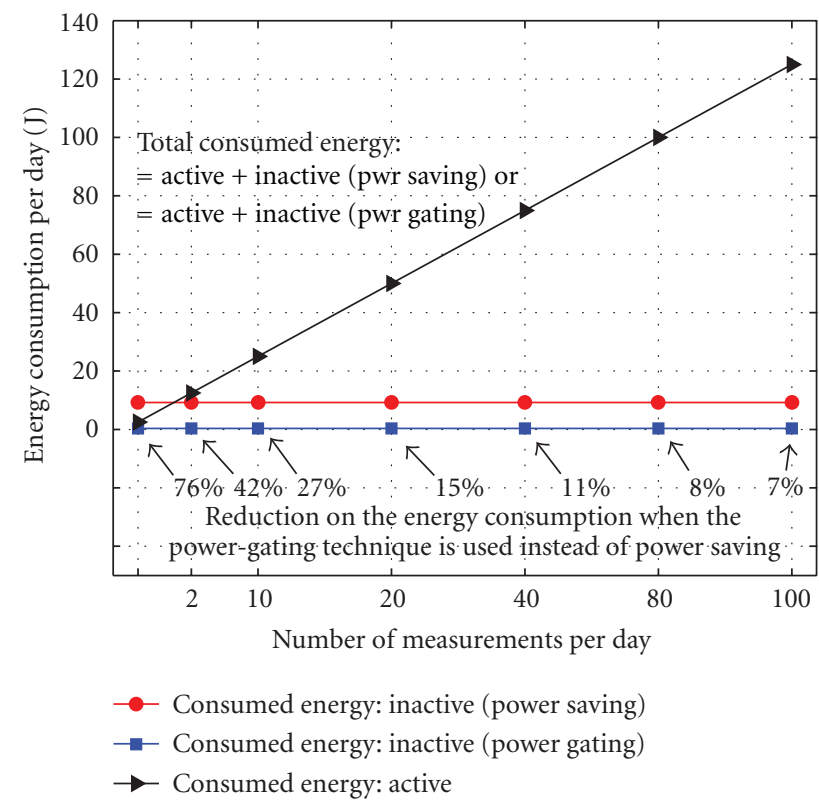

FIGURE 2: Energy consumption of a WSN node in an ideal scenario for different application duty cycles. Modules not being used are disabled using two techniques: power-saving and power-gating.

of the active and the inactive (power-saving) values. For the power-gating mode, similarly it is the sum of active and the inactive (power-gating) values.

As shown in Figure 2, as the frequency of measurements increases, the active energy also increases, as expected. Similarly, the inactive mode energy, corresponding to the intervals when the node is sleeping or has some of its modules disabled, must decrease when the number of cycles increases. Although this is true, Figure 2 does not clearly show this fact because such variations are very small compared to the active energy. Such observation also give us the intuition that the proposed PG technique does have a great impact on the energy when the duty-cycle goes beyond a certain point.

The most important aspect to highlight in this simulation is the significant energy reduction achieved with the power-gating technique. When an extremely low duty-cycle is considered, such as $0.02 \%$, which corresponds to 2 measurements per day in Figure 2, such reduction is close to $76 \%$. As the application duty-cycle increases, the advantages of the power-gating technique becomes less pronounced. For instance, for 100 measurements per day (1.16\% duty-cycle), the energy consumption is only reduced by $7 \%$.

Nonetheless, such low duty-cycle sense-send-sleep applications are not rare cases. Soil moisture measurements $[5,6]$ and antimold or similar solutions to be installed inside the walls of a building are some of such applications. Even when a higher sampling rate is still required, multiple sensors located at the same physical area can properly divide the workload at the time domain resulting in an individual low duty cycle. Although the concept of power-gating is not a novelty, none of the current off-the-shelf WSN nodes use such approach, with the exception of the Waspmote [7]. In the next section, we provide the explanations for this fact in conjunction with design guidelines.

2.2. Leakage Gating and WSNs. We investigated the possibility of adopting the power-gating technique on typical offthe-shelf WSN nodes. The process is not simple because the complexity of the hardware and software significantly increases. For instance, due to the high time delay to effectively activate the radio, the current WSN communication protocols are typically impacted by the PG technique. After designing the proper software modules, the proposed technique proved to be feasible. By using external analog switches, the expected energy achievements shown in Figure 2 are confirmed.

Another factor that increases the complexity of the solution is the fact that not only power lines are associated with leakage: if two modules are connected, such as MCU and radio transceiver, any interface line between these modules can potentially be a leakage point even when one of them 
is completely powered off. The issue can occur with $\mathrm{I} / \mathrm{O}$ lines and ADC channels. Typically, the interfaces lines must be empirically measured, one-by-one, in relation to the existence of leakage currents. To reach this goal, special lowpower instrumentation is necessary. In our work, we evaluated the leakage influence of each MCU line using the special instrumentation in order to detect current variations on the order of nanoamperes [8].

Once a line with significant current leakage is detected, an additional analog switch can be added to that circuitry. We call such technique leakage gating, as an extension for the power-gating not restricted to power lines. Unfortunately, the choice of a PMOS or an NMOS transistor may not be straightforward as in the case of power lines. It is necessary to carefully verify the effects of the switch to the circuitry. Switch parameters such as $R_{\text {DS_ON }}$ may be not so important in leakage gating as it is for a radio transceiver power gate. In many cases, a less expensive analog switch can be effectively used for leakage gating.

Nonetheless, the most important parameter to consider for the switch is its own current leakage $\left(I_{\mathrm{LEAK}}\right)$, also called gate leakage. Ideally, this current must be a small fraction of the leakage current which is expected to be controlled. In our experiments, we investigated 21 different types of switches and we opted for the use of a single optobased one with $I_{\text {LEAK }}<50 \mathrm{nA}$ and $R_{\mathrm{DS}_{-} \mathrm{ON}}<80 \mathrm{~m} \Omega$.

As expected, with the addition of more switches, the complexity of the overall system significantly increases because its proper operation depends on the correct sequence and timing to switch on/off multiple leakage gates. In some cases, power lines and other correlated lines can be switched by a single pin/command. However, in other cases, the timing sequence is required, aggravating even more the time delay necessary for the full operation of the system. In fact, in our experiments using off-the-shelf MCU and radio transceiver, the required time delay reaches values as high as $0.5 \mathrm{~s}$. Therefore, such leakage-control technique cannot be used in WSN nodes without substantial modification of the existing network software.

Fortunately, most I/O lines are connected to high impedance loads and the associated leakage is negligible, not justifying the leakage-gating switch. However, it was not the case for one the transceiver modules we tested: XBee-Pro (MaxStream Inc.). The reception line of this module has a leakage current of around $30 \mu \mathrm{A}$, more than 40 times the overall sleeping current of our final implementation of the node. As already explained, for very low duty-cycle applications (e.g., <0.5\%), such additional loss is significant and we finally opted by using a switch for this line.

\section{Avoiding Voltage Regulators}

Voltage regulators are typically used for the proper operation of radio transceivers and sensor modules in WSN nodes. The energy efficiency of some of these regulators is very impressive and reaches values higher than 95\%. Therefore, the inclusion of such regulators in the design of power modules for WSN nodes is a well-known practice. With an increasing

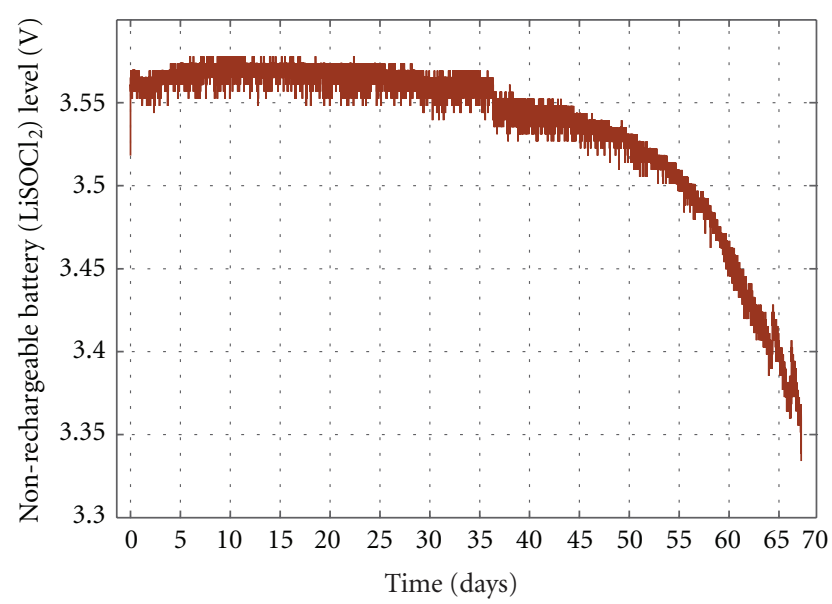

Figure 3: Battery level discharging profile. Experiment with a typical WSN node taking measurements and sending data every minute (17 dBm $\left.T X_{\text {pwr }}\right)$.

emphasis on energy harvesting, rechargeable batteries usually are the main choice for energy storage. In this scenario, a voltage regulator for the processor (MCU) is typically a requirement due to the dynamic range of the voltage level of the cells and also to optimize the usage of the energy remaining at the batteries. Once we turn our attention to nonrechargeable batteries, it is not clear in the WSN literature if the discharging behavior of such primary cells also requires a voltage regulator for the MCU. In this section, we will demonstrate that in general this is not the case and the avoidance of such regulators for powering the MCU is an option to be evaluated.

If we temporarily disregard the effect of pulse currents, a topic to be considered in Section 4, the voltage level of a primary cell has a small variation during the lifetime of the cell when compared with a rechargeable cell. For instance, we intensively used in our experiments high-capacity/lowcurrent lithium thionyl chloride $\left(\mathrm{LiSOCl}_{2}\right)$ cells [9]. With an initial voltage level of around $3.65 \mathrm{~V}$, the cell reaches its end of life with a voltage level of $3.33 \mathrm{~V}$, as shown in Figure 3. Observe that such variation is typically acceptable for the majority of low-power MCUs and processors available in the industry. Therefore, no voltage regulator is required for this specific module.

The long-term experiment in our work related to Figure 3 involves node performing and transmitting soil moisture measurements (3 distinct sensors) every minute. Such high duty cycle was used to speed up the experiment. We can observe that the behavior of the battery is very stable. Also, we can infer that in a typical WSN application with 15-min cycle the battery used here would last more than 2 years. In this controlled laboratory experiment, the MCU is directly connected to the battery and the power-gating technique is used. A single-size-D ER34615 (3.6 V), 19 Ah cell is used in a WSN node with a power profile similar to the one in Table 1. To avoid influence from the instrumentation, all sensing and battery data are collected via wireless. 
In contrast with a primary cell, the dynamic voltage range of a rechargeable cell is critical enough to require a voltage regulator for the MCU, as shown in Figure 4. In Table 1, we consider that the power consumption of the MCU in sleep mode is only $2 \mu \mathrm{W}$. Although this value is in agreement with the datasheet of many low-power MCUs, it is important to highlight that no voltage regulator is being considered in that scenario. If the regulator is included, only the power consumption of this component would typically be 1 or 2 orders of magnitude higher than the sleeping power consumption of the MCU. In short, while in sleep mode, the regulator typically dominates the power budget of the node. It is important to highlight that in the specifications of WSN nodes usually the sleeping power is given without adding the contribution of a voltage regulator. Unfortunately, due to the crescent focus on harvesting systems for WSN nodes, typically the voltage regulator for the MCU is always present and such approach is repeated in many WSN designs even when the node is powered by primary cells.

The avoidance of voltage regulators can be summarized as an effort to use MCUs/processors that support the voltage level variation of a nonrechargeable battery during all the lifetime of the cell. In our studies, we figured out that the implementation of this technique is relatively easy to be achieved. Even when voltage regulators are still required, such as the ones used for radio transceiver and for ADC measurements (analog sensors), the power-gating technique can be promptly used and these regulators will not contribute significantly to the power consumption of inactive devices.

The main drawback of such technique is the potential reduction of reliability of the system. For the solution related to the Figure 3, a careful design is considered. More specifically, it is very important to monitor if an expected low battery level can cause problems to the modules of the node, that is, to the stability of the system. For instance, in our first design, we used an external real-time clock (RTC) chip that presented problems when the battery level was close to its end-of-life. That problem was not promptly identified based on the datasheet information but we eventually solved the issue by selecting a different model for the RTC device.

A second design limitation for the proposed approach is to maintain all loads (i.e., MCU, sensor(s), radio transceiver), directly or indirectly connected to the battery system, under low-power level. For instance, due to the absence of a voltage regulator, a short high-current pulse from any load may restart the MCU. However, radio transceivers and sensors modules can potentially have an intermittent high-power profile. Moreover, when a low-current non-rechargeable battery drives high-current pulses, the lifetime of the cell is drastically reduced [10]. Unfortunately, it is not possible to accurately obtain such information from the battery's datasheet [9]. In our outdoors experiments, we faced lifetime reductions varying from 50 to $90 \%$. Although high-power primary cells are available, they are expensive and their energy capacity are still drastically reduced. We tested 9 different models of $\mathrm{LiSOCl}_{2}$ cells and concluded that a lowcurrent model [9] can still be effectively adopted for WSNs. However, an additional technique is required to extend the

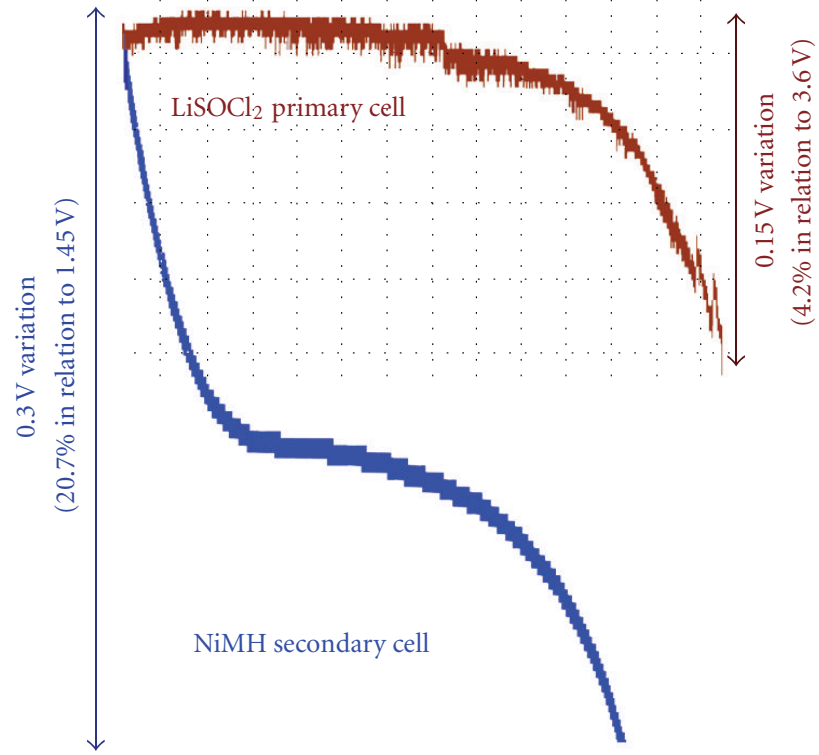

FIGURE 4: Typical-normalized discharging curves for primary and secondary cells in WSN nodes.

lifetime of the system which is subject to high-current pulses. This aspect is considered in the next section.

So far, the proposed power-management techniques considered a scenario where the WSN nodes operate in very low duty-cycles and with primary cells. The main goal behind those techniques is to reduce the active and inactive power consumption as much as possible. By inactive power, we are referring to the intervals when the module is not being used, in special when the node is sleeping. However, intermittent high currents from the sensors and radio modules are expected, as shown in Table 1, and can potentially decrease the lifetime of the primary cells. Therefore, the third technique presented in this section is not exactly a way to reduce the energy consumption, but a low-cost strategy to avoid the reduction of the nominal energy capacity of a primary cell.

The fact that the nominal lifetime of a primary cell can be affected by pulse currents [10] can be confirmed by analyzing the Table 1 in conjunction with Figure 3. Assume a 15minute sampling rate, a total sleeping power of $4 \mu \mathrm{W}$, and that the first 2 mentioned techniques are in use. Therefore, the energy consumption for each cycle is around $1,114 \mathrm{mWs}$. For a $19 \mathrm{Ah}$ primary cell and $3.53 \mathrm{~V}$ average voltage level, the theoretical maximum lifetime of the system is 2,258 days. Compare this value with 1,005 days extrapolated from Figure 3 for a 15 -minute sampling rate $(67 * 15)$. Therefore, a lifetime reduction of around $55 \%$ is expected even for a scenario where the temperature, self-discharging, and battery aging effects are highly minimized. In fact, based on the datasheet of the manufacturers [9], intermittent highdischarging currents from low-current primary cells can extremely reduce the lifetime of the cell (pulse current effect). 


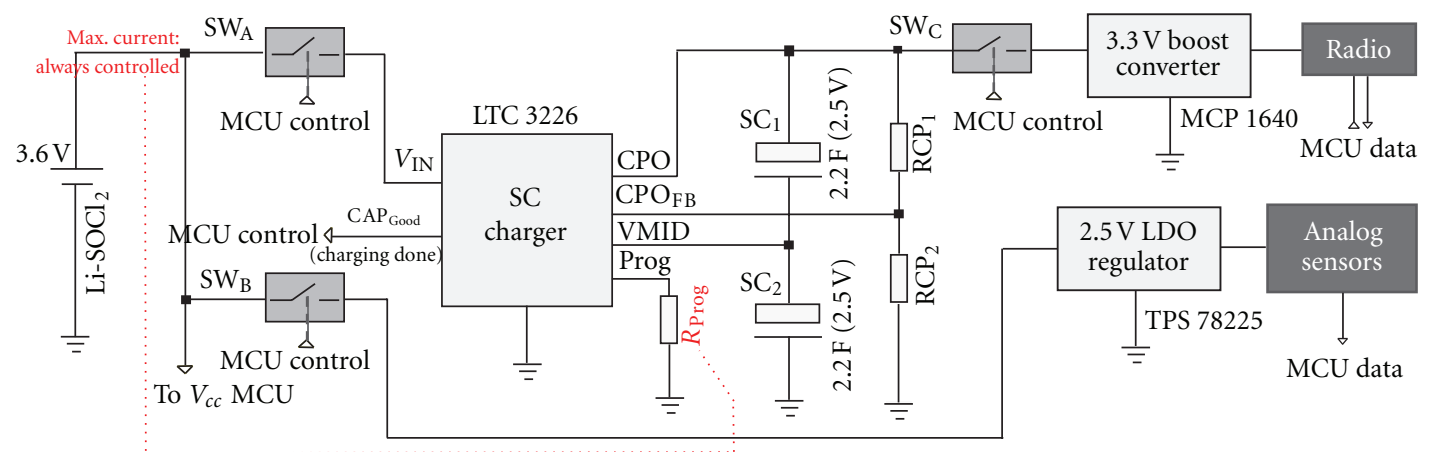

FIGURE 5: Basic power-matching circuitry for a typical WSN node: intermittent high-power load (bold lines) with a low-current/high-capacity nonrechargeable battery.

\section{Power-Matching Technique}

As a rule of thumb, the higher is the energy capacity of a cell, the smaller is its power capacity. For instance, an alkaline battery can provide higher currents but its energy capacity is many times smaller than the energy of a $\mathrm{LiSOCl}_{2}$ cell for the same physical volume. For a very long lifetime, a low-current profile cell (i.e., high energy capacity) is usually the proper option. Due to the pulse current effect, a power matching technique is proposed in order to allow the use of continuous low currents no matter if high-power loads are intermittently activated.

The typical component used for power matching is the supercapacitor (SC) [11]. A longer time to charge the SC (low-current charge) is traded in favor of a shorter highcurrent discharge. The smaller is the charging current, typically limited to $5-35 \mathrm{~mA}$ for $\mathrm{LiSOCl}_{2}$ cells, the longer is the charging process. By controlling that the maximum current never goes beyond the recommended nominal, continuous battery current as defined by the cell manufacturer, the mentioned pulse effect does not occur. However, as previously mentioned, a significant time delay (e.g., seconds to minutes) is introduced due to the need of slow-charging the $\mathrm{SC}(\mathrm{s})$. As a result, such technique cannot be effectively employed for WSNs or any other communication scenario where high data rate and low data delays are required. Moreover, the underlying network protocols must be very efficient and highly deterministic: the usage of the radio transceiver must be short and well defined.

Our proposed model for a power-matching circuitry is shown in Figure 5. This configuration is highly optimized for WSN nodes while maintaining relative low hardware complexity. The first aspect to consider in this model is the configuration for the SCs. The typical maximum voltagelevel range for SCs $(2.5$ to $2.7 \mathrm{~V})$ is usually not sufficient to power WSN radios. Even adopting a boost-converter between the SC and the radio, the operation of the converter would be mainly on a region with small energy efficiency. Therefore, 2 SCs connected in series is proposed as the ideal circuitry for this scenario. Although the effective capacitance decreases by $50 \%$, the autodischarging issue is highly minimized, as explained latter in this section. Observe that the power-gating technique is applied for both SC charger and the radio transceiver. The SC charger can be simply implemented with a resistor. However, in order to reduce the charging time and increase the efficiency of the charging process, off-the-shelf solutions are recommended. In our experiments, we opted by using a commercial 2-cell SC charger (LTC3226 [12]).

Besides the introduction of a significant time delay, the power-matching circuitry also presents inefficiencies in terms of energy. There are multiple causes of losses even for the relative simple circuitry shown in Figure 5. These losses are very related to each other and also with the characteristics of the application. Therefore, a simplified (worstcase scenario) and systematic design approach is proposed in this section specifically for low-date rate and sense-sendsleep WSN applications.

Three Techniques Combined. As shown in Figure 5, the 3 techniques are combined for our final solution. The powergating is achieved with 3 analog switches. Omitted for clarity, additional switches are used for some external lines of the MCU (leakage-gating). Note that the MCU is directly connected to the battery, that is, no voltage regulator is used for the MCU. Finally, 2 SCs, the SCs charger, and a $3.3 \mathrm{~V}$ boost-converter represents the power-matching circuitry.

Overall Strategy. Our ultimate goal is to verify if the total energy losses due to the power-matching technique is still smaller when compared with the loss due to the pulse current effect. Accordingly, a systematic approach is proposed in Figure 6. Next, we follow this approach using specific design options as a complete example.

Steps 1 and 2. In our case, the maximum voltage across the 2 SCs in series is $5 \mathrm{~V}$ because we used $2.5 \mathrm{~V}$ SCs. Sometimes, this value is limited by a smaller available battery level. This is not our case because SC charger we selected (LTC3226) has a charge pump: it charges SCs from 2.5 to $5.5 \mathrm{~V}$. Therefore, in this example, the maximum SC voltage $\left(V_{\mathrm{SC}}^{\max }\right)$ is constrained by $5 \mathrm{~V}$. Next, we are interested in finding the optimum value for $V_{\mathrm{SC}}^{\max }$ and the answer is directed related to the choice of the voltage converter. We selected the MCP1640 3.3 V boostconverter [13] and based on its empirical performance we 


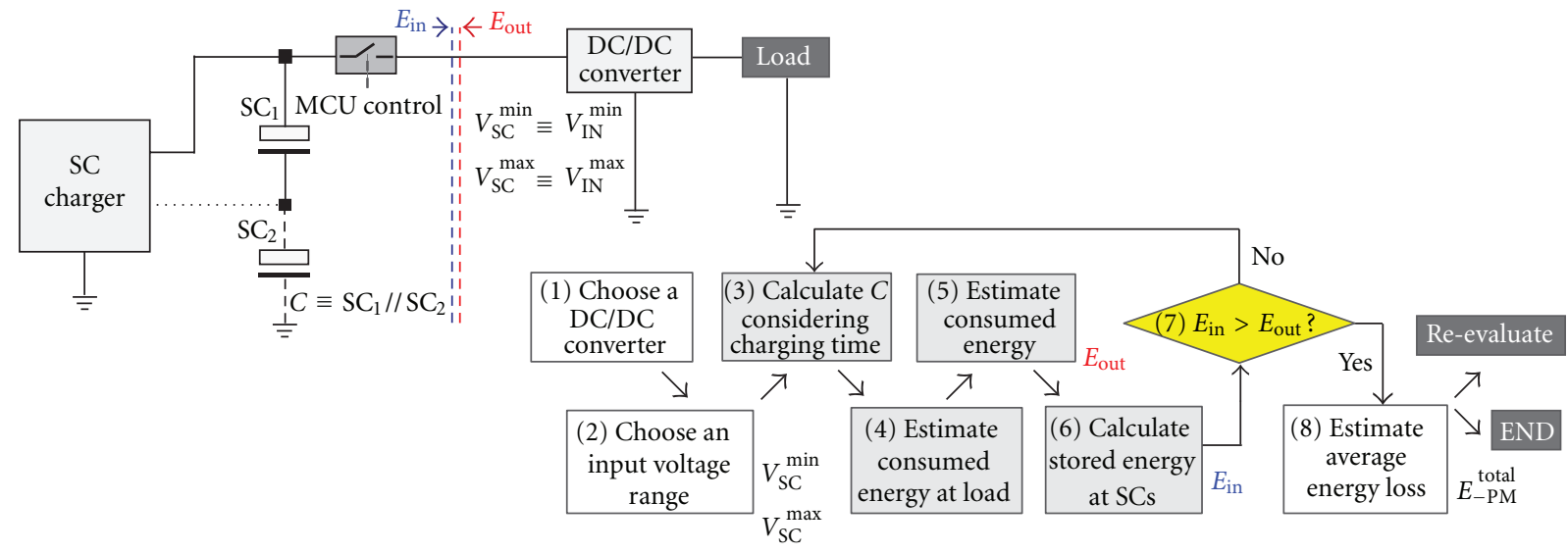

FIGURE 6: Strategy for the power-matching circuitry design.

defined its dynamic input voltage range as $1 \mathrm{~V}\left(V_{\mathrm{SC}}^{\mathrm{min}}\right)$ to $3 \mathrm{~V}$ $\left(V_{\mathrm{SC}}^{\max }\right)$. The latter value can be adjusted by $\mathrm{RCP}_{1}$ and $\mathrm{RCP}_{2}$ shown in Figure 5.

Step 3. A small value of $C$ may be not sufficient to store the required energy for the load. However, a higher $C$ may require an excessive longer time for the SC charge. In this step, we would like to select a high value for $C$ associated with a reasonable time delay for the application. The theoretical maximum time to charge $2 \mathrm{SCs}$ in series, each one with capacitance $C$ is $5 R(C / 2)$, where $R$ is the virtual series resistance of the charging system. We just need to evaluate the worse case for this $R$, as expressed next. According to the final SC charging implementation, the charging time can be only a fraction of what we are estimating in this step. Considering our primary cell, we opted by a maximum charging current of $35 \mathrm{~mA}$ ( $I_{\text {charge }}^{\mathrm{max}}$ ) which can be adjusted by $R_{\text {PROG }}$ shown in Figure 5. Assuming a maximum voltage of $3 \mathrm{~V}\left(V_{\mathrm{SC}}^{\max }\right), T_{\text {charge }}^{\max }$ is given, in seconds, by

$$
T_{\text {charge }}^{\max }=5 \frac{\mathrm{VSC}_{\max }}{I_{\text {charge }}^{\max }}\left(\frac{C}{2}\right)=2.5 C \frac{3}{.035} \cong 214 C .
$$

In our design, we finally selected $C=2.2 \mathrm{~F}$, thus, $T_{\text {charge }}^{\max }=$ $470 \mathrm{~s}$. However, we empirically determined that, for completed discharged SCs, $T_{\text {charge }}^{\text {real }}=165 \mathrm{~s}$. Moreover, if the SCs are not completely discharged, which is usually the case, this time is much smaller. Nonetheless, it is already clear that if we opted for $C=100 \mathrm{~F}, 15$-minute cycle WSN application would not be feasible. Therefore, establishing reasonable boundaries for $C$ is the goal of this step.

Step 4. We want to store sufficient energy in the SCs for properly powering the load for a certain time. However, an excessive nonused energy remained in the SCs represents an important loss (i.e., autodischarge) that we want to minimize. To this end, a worst-case scenario for the load behavior is defined and the maximum consumed energy at the load $\left(E_{\text {load }}^{\max }\right)$ is estimated accordingly. In our case, we concluded that in the event of a communication failure, an additional transmission must be supported. In other words, the communication time must double, from 3 to $6 \mathrm{~s}$. Therefore, $E_{\text {load }}^{\max }$ is estimated as follows:

$$
E_{\text {load }}^{\max }=P_{\text {load }} * \text { Time }_{\text {active }}=(355 \mathrm{~mW})(6 \mathrm{~s})=2.13 \mathrm{~J} .
$$

Step 5. Sometimes, it is necessary to empirically determine the average efficiency of the converter $\left(\eta_{\text {conv }}^{\text {avg }}\right)$ for the entire input voltage range defined at Step 2. Alternatively, the information from the converter datasheet can be used to extrapolate $\eta_{\text {conv }}^{\text {avg }}$. We used the second approach to estimate $\eta_{\text {conv }}^{\text {avg }} \cong 85 \%$. Small errors in this estimation can be corrected latter by adjusting the value of $C$ (thus, $T_{\text {charge }}^{\max }$ also). Therefore, the maximum effective energy required from the SCs $\left(E_{\text {out }}\right)$ is given by

$$
E_{\text {out }}=\frac{E_{\text {load }}^{\max }}{\eta_{\text {conv }}^{\text {avg }}}=\frac{2.13}{0.85}=2.5 \mathrm{~J} .
$$

Step 6. From Step 3, a value for $C$ was already selected. The associated energy stored $\left(E_{\text {in }}\right)$ at the 2 capacitors, as a whole, is given by

$$
E_{\text {in }}=\frac{1}{2} C_{\text {eq }}\left(V_{\mathrm{SC}^{\max }}^{2}-V_{\mathrm{SC}^{\min }}^{2}\right)=\frac{1}{2}(1.1)\left(3^{2}-1^{2}\right)=4.4 \mathrm{~J} .
$$

Step 7. $E_{\text {in }}$ must be higher than $E_{\text {out }}$, otherwise, we must return to Step 3 and choose a higher value for $C$ (thus, increasing the time to charge the SCs). In this example, the goal is satisfied even if we consider a variation of the value of $C$ as high as $40 \%$. A relative high gap between $E_{\text {in }}$ and $E_{\text {out }}$ is usually necessary because the temperature and fabrication process can cause fluctuations on the value of $C$.

Step 8. At Section 3, we figured out that lifetime of the primary cell is reduced by $55 \%$. It means that, for a $19 \mathrm{Ah}$ $3.6 \mathrm{~V}$ cell, the pulse current effect causes an energy loss of $135 \mathrm{KJ}$. If during the battery's lifetime, the losses due to the power-matching circuitry becomes closer to this value, the technique loses its attractiveness. In this step, we want 
to estimate these losses ( $\left.E_{-\mathrm{PM}}\right)$ to confirm if the powermatching technique is an option. One can empirically determine with accuracy $E_{-\mathrm{PM}}$, but this approach is not practical for a scenario involving multiple years. An alternative is to estimate the worst case $\left(E_{-\mathrm{PM}}^{\max }\right)$ : assume that all the energy at the SCs related to the voltages 0 to $V_{\mathrm{SC}}^{\mathrm{min}}$ are completely lost due to the autodischarge of the SCs. Therefore, if we assume that the discharge occurs as soon as the SCs are charged, $E_{-\mathrm{PM}}^{\mathrm{max}}$ is given by

$$
E_{-\mathrm{PM}}^{\max }=\frac{1}{2} C_{\mathrm{eq}} V_{\mathrm{SC}^{\min }}^{2}=\frac{1}{2}(1.1)\left(1^{2}\right)=0.55 \mathrm{~J} .
$$

In our experiments, we actually verified that $E_{-\mathrm{PM}}<0.2 \mathrm{~J}$ for each cycle, on the average. In fact, two factors contribute to the low autodischarge effect in this scenario. First, the value of $C$ is relatively small and such effect is minimized. Second, although the maximum voltage at each $\mathrm{SC}$ is $2.5 \mathrm{~V}$, the actual value is never beyond $1.5 \mathrm{~V}\left(V_{\mathrm{SC}}^{\max } / 2\right)$. The closer the voltage level is to the upper limit, the higher the autodischarging. However, we still need to estimate the cumulative losses for all cycles $\left(E_{-\mathrm{PM}}^{\mathrm{total}}\right)$, which is given by (for 15-minute cycles):

$$
E_{-\mathrm{PM}}^{\mathrm{total}}=N_{\text {cycles }} * E_{-\mathrm{PM}}^{\mathrm{max}}=\frac{1005 \text { days }}{15 \mathrm{~min}}(0.55)=53 \mathrm{KJ} .
$$

Even with a conservative approach, the power-matching technique will reduce at least $60 \%$ of the pulse current effect on primary cells. However, if this technique is found not to be an option, multiple cells in parallel is a simplistic solution that can potentially mitigate the mentioned issue. The tradeoffs of this alternate option are the higher cost and higher physical volume of the node.

\section{Comparison with Typical Power Management Approaches}

Due to the fact that some of the techniques presented in this paper have been used in the industry in different scenarios, the goal of this section is to highlight the contributions of our work in particular for solutions based on WSNs.

Power-Gating Aspects. Although is a well-known technique, for the best of our knowledge, we present the first real-world implementation of the PG technique for a WSN node also associated with the extension of this technique, called leakage gating (LG). This latter technique is the application of the PG concept to any other line that presents significant current leakage when the node is in sleep mode. An important tradeoff of the PG/LG techniques is the larger form factor (and higher cost) introduced with the additional power/signal switches. However, because the total cost of a WSN node is also associated with its support, the ultimate question behind the PG/LG is if the higher cost of a node justify the expected costs reduction associated to the frequent need of exchanging batteries. For the antimold example mentioned in this paper, the higher deployment cost due to PG/LG seems to be justified. The same for a node deployed in an area of difficult (or expensive) access. However, for certain cases, the technique may not be justified or, at least, just a small number of PG/LG switches can be realistically important to save energy.

Regulator Avoidance Aspects. The proposed technique in this paper is specifically associated with the main MCU and its RTC, if available. In a WSN node, these two components must be continuously connected to a power source when the node is sleeping/hibernating. If a voltage regulator is used in series with the power source, such regulator will always contribute for the sleeping power of the WSN node. Therefore, by eliminating this components we are minimizing the total sleeping power of the node. We highlighted in this paper that such approach is potentially feasible with primary cells but not with secondary cells. Nonetheless, the ultimate application of this technique is dependent on the choice of the primary cell, MCU, and RTC components of the WSN node. For instance, if the maximum supply voltage for an ultra-low-power MCU is $1.8 \mathrm{~V}$, it is clear that the inclusion of a voltage regulator is a wise decision if a $3.6 \mathrm{~V}$ primary cell is involved. With other chemical types of cells, different voltage levels are provided and 3.6 V must not be considered a fixed value for the mentioned technique. If the technique is expected to be used, it is important to find the proper battery type/configuration that is best tailored to the MCU and RTC.

Supercapacitor Charger. At the time this paper was written, there were few off-the-shelf SC charger chips available. However, the efficiency of the charger device used in our work clearly justifies its adoption rather than designing the circuitry for this task. In particular, the chip we used in our implementation (LTC3226 from Linear Circuits, Inc.) takes care of the charge balance between the SCs without the risk of having a voltage across an SC which is higher than its nominal maximum voltage.

Quiescent Current of a Voltage Regulator. When considering the voltage regulator avoidance technique, one can highlight that modern regulators have a very small quiescent current (e.g., $<1 \mu \mathrm{A}$ ) which prevents the adoption of the technique. Such argument is potentially valid in relation to the PG technique. For instance, if we assume that the $2.5 \mathrm{~V}$ LDO in Figure 5 can be disabled and still maintain a very low quiescent/shutdown current, then the PG switch SW_B is not justified. However, if we consider the argument again for the voltage regulator technique, it is rarely valid. The goal of this technique is to connect the MCU (and/or RTC) components directly to the power source, such as a nonrechargeable battery in order to reduce the sleeping power of the node. Typically, the quiescent current in the datasheet of a voltage regulator assumes no load or, in some cases, the complete deactivation of the regulator. However, because the MCU still requires a small current while sleeping, the nominal value of the quiescent current may not be applied in this case and tests must be conducted. On the other hand, if an active regulator still has high efficiency (e.g., $>80 \%$ ) while supplying very small currents to MCU and RTC modules (the former in sleep mode), then the argument holds and the adoption of the voltage regulator is justified. Therefore, such aspect must 
TABLE 2: Overview of the proposed power management techniques.

\begin{tabular}{|c|c|c|c|c|c|}
\hline Technique & Costs & $\begin{array}{c}\text { Software } \\
\text { complexity }\end{array}$ & $\begin{array}{c}\text { Intrinsic energy } \\
\text { inefficiencies }\end{array}$ & $\begin{array}{c}\text { Main } \\
\text { trade-off }\end{array}$ & $\begin{array}{c}\text { Usage } \\
\text { indication }\end{array}$ \\
\hline Power gating (PG) & Add $+10 \%$ & High & Very low & High data latency & $\begin{array}{c}\text { Very low } \\
\text { duty-cycle app }\end{array}$ \\
\hline $\begin{array}{l}\text { Voltage regulator } \\
\text { avoidance }\end{array}$ & None & None & $\begin{array}{l}\text { MCU w/high volt. } \\
\text { can be } \\
\text { energy-inefficient }\end{array}$ & $\begin{array}{l}\text { MCU can be } \\
\text { unstable }\end{array}$ & $\begin{array}{c}\text { Primary cells + low } \\
\text { duty-cycle app }\end{array}$ \\
\hline Power matching (PM) & Add $+15 \%$ & High & $\begin{array}{c}\text { May have }+30 \% \\
\text { energy losses }\end{array}$ & High data latency & $\begin{array}{c}\text { Primary cells + low } \\
\text { duty-cycle app }\end{array}$ \\
\hline
\end{tabular}

be evaluated case-by-case. In our implementation, we did not find a regulator which satisfied the mentioned requirements and it was not adopted, as shown in Figure 5.

Reducing Operating Voltage of the MCU. A technique so far not mentioned in this work, but highlighted by the processors/MCUs manufacturers, is the reduction of the operating voltage. Typically, this approach is also associated with a smaller clock speed. Nonetheless, such technique basically reduces the power consumption while the device is in active mode. In general, the technique does not provide significant advantages when the processor is in sleep mode. In other words, if low duty cycle applications are not assumed, the adoption of this technique is strongly recommended. That is, a value such as $5 \mathrm{~mW}$ in Table 1 , could be reduced to smaller value, such as $1 \mathrm{~mW}$. On the other hand, the adoption of a smaller MCU voltage may require a voltage regulator between the power source and the MCU. Because such device can introduce significant loss when the MCU is sleeping, there is a minimum application duty-cycle that supports the adoption of the technique. In other words, the mentioned voltage reduction technique would decrease the height of the line Consumed Energy: active in Figure 2 but also would increase the height of the other two lines (inactive) due to the additional losses caused by the introduction of a voltage regulator for the MCU. For the context of this work (low duty-cycle applications) and the selected components used in our sensor node implementation, the reduction of the operating voltage of the MCU was not considered a proper option.

Real-World Implementations in WSN Nodes. to the date, there is no report about a WSN node that implements the combined techniques mentioned in this work. The Waspmode device (Libelium Comunicaciones Distribuidas S.L.) [7] implements PG switches for multiple modules. Even related to the avoidance of a voltage regulator, the models of WSN modules that target rechargeable batteries, employ a voltage regulator for the MCU. In our work, we designed three different WSN nodes: Ripple-2A, Ripple-2D, and Ripple-2D+. The first one is based on rechargeable batteries and solar panel (Figure 1 and implements the PG technique in 4 modules and also the regulator avoidance technique. The second device is based on nonrechargeable battery $(3.6 \mathrm{~V})$

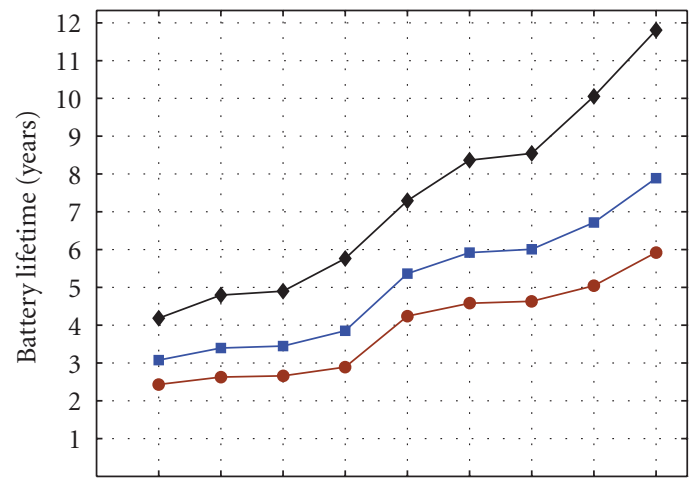

Technique 1 Off Off On On Off On Off On On (empirical) Technique 2 Off On Off On On Off On On On (empirical) Technique 3 Off Off Off Off On On On On On (empirical)

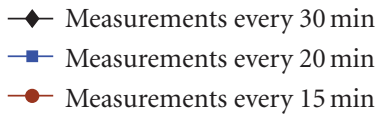

FIGURE 7: Lifetime of a WSN node using a 19 Ah battery according to different power techniques.

and implements the PG and regulator avoidance techniques. The third node, the default node for our real-world deployments, implements the three techniques analyzed in this paper. The first deployment (Matthaei Bothanical Gardens, Ann Arbor, MI) was realized on August 2011 and involved 26 sensor nodes. The second deployment (Canton, OK) was realized on September 2011 and involved 21 nodes. The third deployment (Sacramento, CA) has started on August 2012 and involves 150 nodes spread in an area larger than $3 \times 3 \mathrm{~km}^{2}$ [14].

This third deployment mentioned above is solely based on Ripple-2D+ which employs PG by means of 4 analog switches PS710-B (NEC Inc.). Beside the Turn-On Time (TOT) due to the switch (up to $5 \mathrm{~ms}$ ), each load has its own TOT characteristic. For instance, we empirically determined that the TOT of the radio transceiver XBee Pro SBP1 (Digi Inc.) is higher than $200 \mathrm{~ms}$. Such value is high enough to prevent the use of the PG technique for the majority, if not all, of the WSN protocols and new networking protocols must be designed considering PG. Similarly, the PM technique can potentially increase the transmit delay because 
$\mathrm{SC}(\mathrm{s})$ must be charged before the realization of a radio communication or similar high-power task. Therefore, a very deterministic network behavior is expected in order to properly design the amount of charge regularly stored in the SCs. In our sensor node implementation, more than $15 \%$ of the code is related to the management of the PM technique. Also, the SC circuitry corresponds to more $15 \%$ of the costs of our sensor node.

\section{Conclusions}

Three power-management techniques are proposed specifically for sensor nodes used in low duty-cycle WSN applications and powered by primary cells. Accordingly, a customized node is designed and both laboratory and outdoor experiments are performed. Semiempirical models are used for the simulations and the results are summarized in Figure 7. Disregarding network aspects, these simulations consider worst-case scenarios and our empirical results show significantly better results. In all cases, we conclude that the lifetime of a node can be strongly extended by multiple folds with the approaches proposed here.

The main trade-offs of the solution are the increase of both data-latency and complexity. The third technique, in particular, imposes operational delays on the order of minutes constraining its application to some communication scenarios. Also, the effects due to aging and extreme temperature variations are not considered in our models. Accordingly, we envision two directions for our future work: the development of novel WSN protocols to take advantage of the proposed techniques and the realization of long-term experiments (multiple years).

In general, only low (or very low) duty-cycle applications can be indicated for these techniques, as shown in Table 2. Nonetheless, we believe that the future of many networks reside in the capability of dynamically changing between multiple operational modes, being low duty-cycle one of these modes. While in this mode, all the power-management techniques discussed in this paper can be potentially can be adopted as hardware modules that can be dynamically activated and deactivated by software making the final WSN node design very flexible for different and temporary needs.

\section{References}

[1] A. R. Silva and M. C. Vuran, "Empirical evaluation of wireless underground-to-underground communication in wireless underground sensor networks," in Proceedings of the 5th IEEE International Conference on Distributed Computing in Sensor Systems (DCOSS '09), Marina Del Rey, Calif, USA, June 2009.

[2] W. Q, P. Angove, J. Buckley, J. Barrett, and G. Kelly, "Design and performance analysis of an embedded wireless sensor for monitoring concrete curing and structural health," Journal of Civil Structural Health Monitoring, vol. 1, pp. 47-59, 2011.

[3] A. S. Weddell, N. R. Harris, and M. N. White, "Alternative energy sources for sensor nodes: rationalized design for long-term deployment," in Proceedings of the IEEE International Instrumentation and Measurement Technology Conference (IMTC '08), pp. 1370-1375, British Columbia, Canada, May 2008.
[4] M. A. Pasha, S. Derrien, and O. Sentieys, "Toward ultra lowpower hardware specialization of a wireless sensor network node," in Proceedings of the IEEE 13th International Multitopic Conference (INMIC '09), pp. 1-6, Islamabad, Pakistan, December 2009.

[5] H. R. Bogena, J. A. Huisman, C. Oberdörster, and H. Vereecken, "Evaluation of a low-cost soil water content sensor for wireless network applications," Journal of Hydrology, vol. 344, no. 1-2, pp. 32-42, 2007.

[6] M. Moghaddam, D. Entekhabi, Y. Goykhman et al., "A wireless soil moisture smart sensor web using physics-based optimal control: concept and initial demonstrations," IEEE Journal of Selected Topics in Applied Earth Observations and Remote Sensing, vol. 3, no. 4, pp. 522-535, December 2010.

[7] "Waspmote," http://www.libelium.com/products/waspmote.

[8] “2400 Sourcemeter," http://www.keithley.com/.

[9] "Primary lithium batteries ER34615 (lithium thionyl chloride)," http://www. batteryspace.com/prod-specs/ER34615BS-R.pdf.

[10] M. Doyle, T. Fuller, and J. Newman, "Modeling of galvanostatic charge and discharge of the lithium/polymer/insertion cell," Journal of the Electrochemical Society, vol. 140, no. 6, pp. 1526-1533, 1993.

[11] C. Menachem and H. Yamin, "High-energy, high-power pulses plus battery for long-term applications," Journal of Power Sources, vol. 136, no. 2, pp. 268-275, 2004.

[12] "LTC3226-2-Cell supercapacitor charger," http://www.linear .com/product/LTC3226.

[13] "MCP1640 Data Sheet," http://www.microchip.com/.

[14] A. R. Silva, Mingyan Liu, and Mahta Moghaddam, "Ripple2: a non-collaborative, asynchronous, and open architecture for highly-scalable and low duty-cycle WSNs," in ACM Intl Workshop on Mission-Oriented WSN (MiSeNet '12), Instanbul, Turkey, August 2012. 

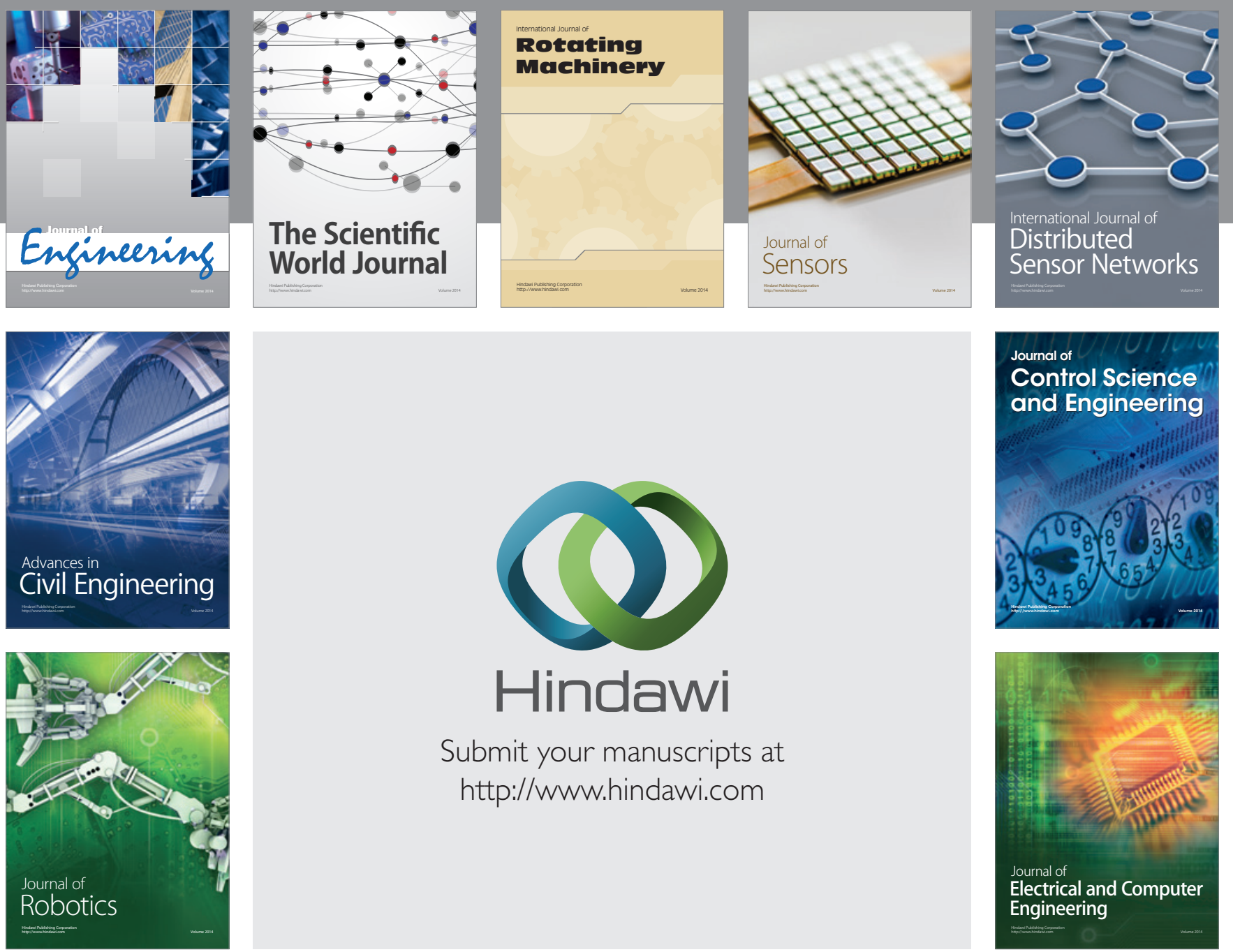

Submit your manuscripts at

http://www.hindawi.com
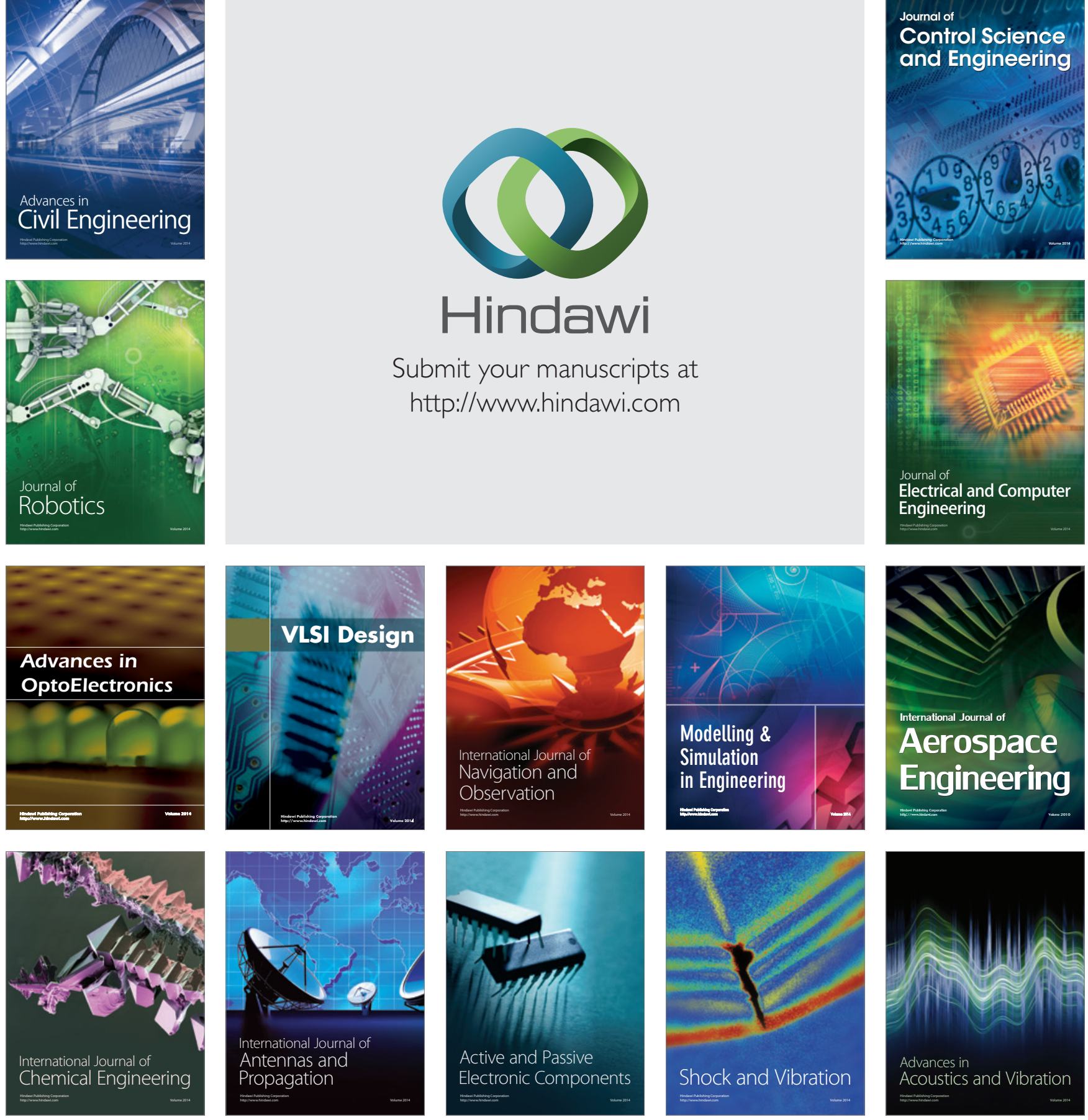